\title{
Magnetic phase evolution and particle size estimation study on nanocrystalline $\mathrm{Mg}-\mathrm{Mn}$ ferrites
}

\author{
K. B. Modi $\cdot$ N. H. Vasoya $\cdot$ V. K. Lakhani $\cdot$ \\ T. K. Pathak
}

Received: 28 October 2013/Accepted: 12 December 2013/Published online: 27 December 2013

(C) The Author(s) 2013. This article is published with open access at Springerlink.com

\begin{abstract}
The nanocrystalline spinel ferrite compositions of $\mathrm{Mg}_{x} \mathrm{Mn}_{1-x} \mathrm{Fe}_{2} \mathrm{O}_{4}(x=0.0,0.2,0.4$ and 0.5$)$ system have been synthesized by the chemical co-precipitation route. The structural and magnetic properties have been studied by means of X-ray powder diffraction (XRD), transmission electron microscopy (TEM) and Mossbauer spectroscopic measurements. ${ }^{57} \mathrm{Fe}$ Mossbauer spectra of three specimens, as prepared, annealed at $200{ }^{\circ} \mathrm{C}$ and sintered at $1,100{ }^{\circ} \mathrm{C}$, of the studied compositions are recorded and analyzed to study the magnetic phase evolution. The Mossbauer spectra of as-prepared samples show a paramagnetic doublet, annealed samples exhibit simultaneous presence of a central paramagnetic doublet superimposed on two broad magnetic sextets while spectra for sintered samples show two well-resolved Zeeman split sextets corresponding to the $\mathrm{Fe}^{3+}$ ions at the tetrahedral sites and the other due to the $\mathrm{Fe}^{3+}$ ions at the octahedral sites of the spinel lattice along with presence of central doublet. The particle size estimated from the probability versus hyperfine magnetic field distribution curve is in agreement with those
\end{abstract}

\section{K. B. Modi ( $\square)$}

Department of Physics, Saurashtra University,

Rajkot 360 005, India

e-mail: kunalbmodi2003@yahoo.com

N. H. Vasoya

Sanjaybhai Rajguru College of Engineering, Morvi Road,

Rajkot 360 003, India

\section{K. Lakhani}

Department of Physics, Bahauddin Science College, Junagadh 362 001, India

T. K. Pathak

Government Engineering College, Kalavad Road,

Rajkot 360 005, India determine from XRD and TEM analysis, validates the method employed.

Keywords Nano ferrite materials - Magnetic properties · Mossbauer spectroscopy

\section{Introduction}

In recent years, magnetic nanoparticles of ferrites have attracted much attention because of their importance in the understanding of the fundamental physical properties as well as their applications in various fields (Singhal et al. 2012). Due to the very small sizes (1-100 nm) novel and/or improved magnetic characteristics are observed for the nanosized magnetic particles when compared to that of the coarse-grained bulk counterpart (Sharma et al. 2005). Nanocrystalline materials can be synthesized either by consolidating small clusters (bottom-up approach) or breaking down the polycrystalline bulk materials into crystalline units with dimensions of nanometer (top-down approach) (Pathak et al. 2010; Vasoya et al. 2010). It is also known that the high temperature sintering of nanoparticle ferrite precursors transforms them into coarse-grained ceramics ferrite materials (Pandya et al. 1991).

In order to characterize nanophase materials Mossbauer spectroscopy can successfully employed to observe the important phenomena of superparamagnetism and collective magnetic excitations. The shape of Mossbauer spectrum depends strongly on the relaxation time $(\tau)$. In the case of ${ }^{57} \mathrm{Fe}$ Mossbauer spectroscopy a magnetically split spectrum with six lines is observed when $\tau>10^{-7} \mathrm{~s}$, where as for $\tau<10^{-10} \mathrm{~s}$, the spectrum consists of one or two sharp lines. But in the intermediate range, the spectra are complex with broad lines. In solid state physics, the analysis of 
the properties of surface layer of nanoparticles is an important but complicated issue. The investigation techniques should make it possible to observe the surface and bulk layers of atoms constituting nanoparticles. Mossbauer spectroscopy provides quantitative information not only on the properties of surface layers but also on the size of nanoparticles (Nikolaev et al. 2001).

Manganese ferrite, $\mathrm{MnFe}_{2} \mathrm{O}_{4}$, is considered as a most controversial spinel ferrite or a complex system (Chen et al. 1996). The distribution of cations among the available tetrahedral (A-) site and octahedral (B-) site, degree of inversion, oxidation state of $\mathrm{Fe}^{3+}$ and $\mathrm{Mn}^{2+}$ and particularly its magnetic properties are still a subject of interest. On the other hand, magnesium ferrite, $\mathrm{MgFe}_{2} \mathrm{O}_{4}$, is a partially inverse spinel and it can be considered as a collinear ferrimagnet whose degree of inversion depends on the thermal history of the sample, microstructure and preparation methodology (Modi et al. 1996). The system under study, $\mathrm{Mg}_{x} \mathrm{Mn}_{1-x} \mathrm{Fe}_{2} \mathrm{O}_{4}(x=0.0,0.2,0.4$ and 0.5), belongs to a large class of compounds having a general formula $\mathrm{A}^{2+} \mathrm{B}_{2}{ }^{3+} \mathrm{O}_{4}{ }^{2-}$ and crystallize in the spinel structure. Due to very high electrical resistivity and low dielectric losses manganese -magnesium ferrites are widely used in microwave devices (Lakshman et al. 2005). The Mg-Mn ferrites are quite versatile from the point of view of their applications and simplicity of their preparation. These ferrites have a rectangular hysteresis loop characteristics making them suitable for the use in memory and switching circuits of digital computers and as a phase shifter (Gagan et al. 2006).

The present work deals with the nanoparticles synthesis of $\mathrm{Mg}_{x} \mathrm{Mn}_{1-x} \mathrm{Fe}_{2} \mathrm{O}_{4}$ spinel ferrite system using the chemical co-precipitation technique, study of magnetic phase evolution as a function of heat treatment and particle size estimation by Mossbauer spectral analysis.

\section{Experimental details}

Nanoparticles of mixed spinel ferrite system: $\operatorname{Mg}_{x} \mathrm{Mn}_{1-x}$ $\mathrm{Fe}_{2} \mathrm{O}_{4}$, with variable compositions, $x=0.0,0.2,0.4$ and 0.5 were prepared by the chemical co-precipitation route. Manganese sulfate $\left(\mathrm{MnSO}_{4} \cdot \mathrm{H}_{2} \mathrm{O}\right)$, magnesium sulfate $\left(\mathrm{MgSO}_{4} \cdot 7 \mathrm{H}_{2} \mathrm{O}\right)$ and ferrous sulfate $\left(\mathrm{FeSO}_{4} \cdot 7 \mathrm{H}_{2} \mathrm{O}\right)$ of analytical reagent (AR) grade were procured from Thomas Baker, Mumbai.

The starting solution was prepared by mixing $50 \mathrm{ml}$ of aqueous solutions of $\mathrm{MnSO}_{4} \cdot \mathrm{H}_{2} \mathrm{O}(0.25 \mathrm{M}), \mathrm{MgSO}_{4} \cdot 7 \mathrm{H}_{2} \mathrm{O}$ $(0.25 \mathrm{M})$ and $\mathrm{FeSO}_{4} \cdot 7 \mathrm{H}_{2} \mathrm{O}(0.5 \mathrm{M})$ in stoichiometric proportions. Deionized double-distilled water was used as a solvent to avoid the production of impurities in the final product. $2 \mathrm{M}$ solution of sodium hydroxide $(\mathrm{NaOH})$ was prepared as a precipitant. The starting salt solution with $\mathrm{pH}$ $\sim 3.5$ was added dropwise to the precipitant because the solubility product constant $\left(K_{\mathrm{sp}}\right)$ for the hydroxides of the cations is exceeded and sequential precipitation of the hydroxides can be avoided. The $\mathrm{pH}$ of the solution was constantly monitored. The reactants were constantly stirred using a magnetic stirrer until a $\mathrm{pH}$ level of 10-11 was reached. A suspension ( $\mathrm{pH}$ 10.5) thus formed, containing dark green intermediate precipitates, was then heated and kept at $60{ }^{\circ} \mathrm{C}$. During heating, hydrogen peroxide $\left(\mathrm{H}_{2} \mathrm{O}_{2}\right)$ was added to promote oxidation reaction until all the intermediate precipitation changed into dark brownish precipitation of the spinel ferrites. The precipitate was then filtered, washed twice with water and then with acetone to remove impurities if any. The precipitate was then dried overnight. The acquired substance was then grinded into a fine powder (as-prepared samples). At this stage the product contains some associated water (up to $10 \mathrm{wt} \%$ ), which was then removed by heating at $200{ }^{\circ} \mathrm{C}$ for $6 \mathrm{~h}$ under vacuum (annealed samples). In order to carry out the structural and magnetic phase evolution study, one set of the as-prepared samples was sintered in air at $1,100{ }^{\circ} \mathrm{C}$ for $18 \mathrm{~h}$ and slowly cooled to room temperature (sintered samples).

$\mathrm{X}$-ray diffraction data were collected using a Philips (PW 1710) automated $\mathrm{X}$-ray powder diffractometer with $\mathrm{CuK} \alpha$ radiation at room temperature $\left(27^{\circ} \mathrm{C}\right)$. TEM images of the samples were taken using TECNAIK 20 (Philips) microscope operated at $200 \mathrm{kV}$. For the TEM observations, the powder was first dispersed in amyl acetate by ultra sonication and then the suspensions were dropped on a copper grid with a carbon film. The grain sizes and shapes were determined from the TEM pictures. Mossbauer spectrometer of electromechanical type with a constant acceleration transducer and a 256 multi-channel analyzer operating in time mode was used to obtain the spectra of the samples at room temperature $\left(27^{\circ} \mathrm{C}\right)$ in transmission geometry and $14.4 \mathrm{keV}$ gamma rays were detected with Xenon-methane filled proportional counter. The radiation source of a $10 \mathrm{mCi}:{ }^{57} \mathrm{Co}$ isotope in a Rh-matrix was used where the absorber thickness was ideally thin $\left(\sim 0.15 \mathrm{mg}{ }^{57} \mathrm{Fe} / \mathrm{cm}^{2}\right)$.

\section{Results and discussion}

The X-ray powder diffraction patterns recorded at room temperature $\left(27^{\circ} \mathrm{C}\right)$ for the as-prepared $(x=0.0,0.2$ and $0.4)$, annealed at $200{ }^{\circ} \mathrm{C}(x=0.2$ and 0.5$)$ and sintered at $1,100{ }^{\circ} \mathrm{C}(x=0.5)$ samples are shown in Fig. 1. The spectra for the as-prepared particles have two broad peaks without any structure. The background noise and broadness of the peaks are characteristic of particles with nanometer dimensions. The X-ray diffractometry showed that annealed samples were single phase spinel. The significant 

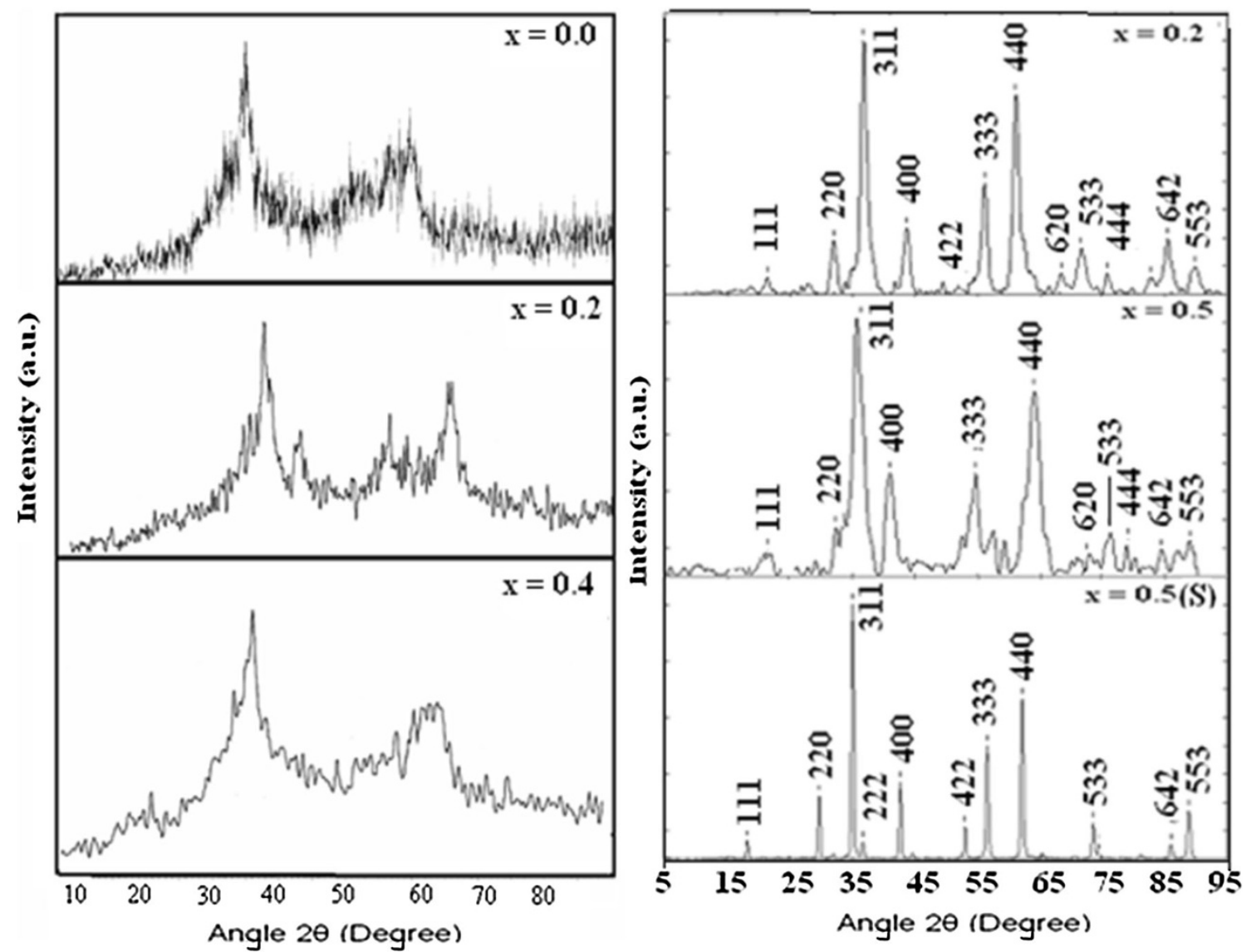

Fig. 1 X-ray powder diffractograms for as-prepared $(x=0.0,0.2$ and 0.4$)$, annealed $(x=0.2$ and 0.5$)$ and sintered (S) $(x=0.5)$ samples of $\mathrm{Mg}-\mathrm{Mn}$ ferrites

line broadening is seen due to nanosized particle formation in annealed samples. This is expected because in the nanosized particles there are insufficient diffraction centers, which causes the line broadening. A representative XRD pattern of sintered sample of $x=0.5$ composition shows sharp Bragg reflections corresponding to the fcc spinel structure; thus, exhibiting no signature of evolution of any extra phase after high temperature sintering, confirming good quality of wet chemically prepared ferrite samples with expected stoichiometry. The sharp Bragg reflections indicate that the fine particle nature is lost due to high temperature sintering and the ferrite materials are akin to the coarse-grained ceramically prepared products.

The cell edge parameter for annealed samples was found to decrease with $\mathrm{Mg}^{2+}$-substitution (Table 1) which is attributed to the replacement of larger cation $\mathrm{Mn}^{2+}$ having Pauling ionic radius of $0.83 \AA$ by smaller cation $\mathrm{Mg}^{2+}(0.66 \AA)$ in the system (Weast 1980-1981). Typical TEM images for the samples annealed at $200{ }^{\circ} \mathrm{C}$ with $x=0.0$ and 0.5 compositions are shown in Fig. 2. It can be seen that particles are quite well dispersed and not much agglomeration is present. The mean particle size for $\mathrm{MnFe}_{2} \mathrm{O}_{4}(x=0.0)$ is found to be $\sim 40 \mathrm{~nm}$, which
Table 1 Lattice constant $(a)$ and average particle size $(D)$ for annealed samples of $\mathrm{Mg}-\mathrm{Mn}$ ferrite compositions

\begin{tabular}{lllll}
\hline Mg-content $(x)$ & $a(\mathrm{~nm}) \pm 0.0002 \mathrm{~nm}$ & \multicolumn{3}{l}{$D(\mathrm{~nm}) \pm 1 \mathrm{~nm}$} \\
\cline { 3 - 5 } & & XRD & TEM & Mossbauer \\
\hline 0.0 & 0.8479 & 38 & 40 & 42 \\
0.2 & 0.8448 & 33 & 36 & 33 \\
0.4 & 0.8427 & 28 & 31 & 30 \\
0.5 & 0.8408 & 25 & 27 & 22 \\
\hline
\end{tabular}

decreases with increasing $\mathrm{Mg}$-content $(x)$ and becomes $\sim 27 \mathrm{~nm}$ for $\mathrm{Mg}_{0.5} \mathrm{Mn}_{0.5} \mathrm{Fe}_{2} \mathrm{O}_{4}(x=0.5)$ composition. It is well known that materials with a cubic crystal structure are prone to grow into a spherical shape to minimize the surface tension (Parekh et al. 2006). TEM images for the typical $x=0.0$ and 0.5 compositions of $\operatorname{sintered}_{(\mathrm{w})}$ product are also shown in Fig. 2 for comparison purpose. However, for sintered samples particles appear in an irregular shape for $x=0.0$ composition, while for $x=0.5$ composition, along with spherically shaped particles with mean particle size of $1.5 \mu \mathrm{m}$, few particles are of irregular shape.







Fig. 2 TEM images for annealed and sintered samples of $x=0.0$ and 0.5 compositions

The average crystallite size was determined from broadening of the high intensity $\mathrm{X}$-ray diffraction line corresponding to (220), (311), (400), (333), (440), (553) planes using the Scherrer's formula (Cullity 1978) incorporating the correction of the instrumental line broadening. The particle size estimated for TEM analysis is slightly greater than the particle size estimated from XRD pattern analysis using Scherrer's formula. This is because X-ray diffraction gives the information of crystalline region only and the contribution from the amorphous grain surface does not contribute. On the other hand, TEM gives the complete picture of the nanoparticles. By analyzing TEM and XRD, one can have almost complete picture of the particle size, their distribution and morphology. Detailed discussion on particle size reduction and change in particle morphology as a function of $\mathrm{Mg}^{2+}$-substitution for $\mathrm{Mn}^{2+}$ in $\mathrm{MnFe}_{2} \mathrm{O}_{4}$ has been given elsewhere (Modi et al. 2012).

${ }^{57} \mathrm{Fe}$ Mossbauer spectra of three specimens, as prepared, annealed at $200{ }^{\circ} \mathrm{C}$ and sintered at $1,100{ }^{\circ} \mathrm{C}$, of $x=0.2$ and 0.4 compositions are displayed in Fig. 3. All the spectra have been fitted using the NORMOS software (Gabriel and Ruby 1965). The solid line through data points is the result of computer fits of spectra obtained assuming equal line widths for the A- and B- sites. The asprepared samples show a paramagnetic doublet, which is due to the superparamagnetic relaxation, that is, each ferrite particle is a single domain. The observed broad doublet in Mossbauer spectrum is indicative of the absence of magnetic hyperfine interaction (long-range ordering). This observation leads to the conclusion that in as-prepared samples of all the compositions, the particles have nano dimension or more probably these ferrimagnetic nanoparticles are separated magnetically from the matrix since the region is surrounded by non-magnetic $\mathrm{Mg}^{2+}$ ions in the system. Thus, the Mossbauer spectra of as-prepared samples reveal the existence of superparamagnetic clusters or paramagnetic centers (Ishikawa 1964). This indicates that ferrimagnetic as-prepared samples are superparamagnetic at room temperature $\left(27^{\circ} \mathrm{C}\right)$, their blocking temperature being lower than the room temperature.

The Mossbauer spectra of annealed samples exhibit simultaneous presence of a central paramagnetic doublet superimposed on two broad magnetic sextets, indicating the partial transformation to an ordered magnetic structure. In other words, the Mossbauer spectra of annealed samples exhibit signature of evolution of long-range magnetic order co-existing with paramagnetic phase. The doublet can only be observed when the superparamagnetic relaxation of the nanoparticles occurs at a rate faster than the Mossbauer measurement time, giving a time average zero 

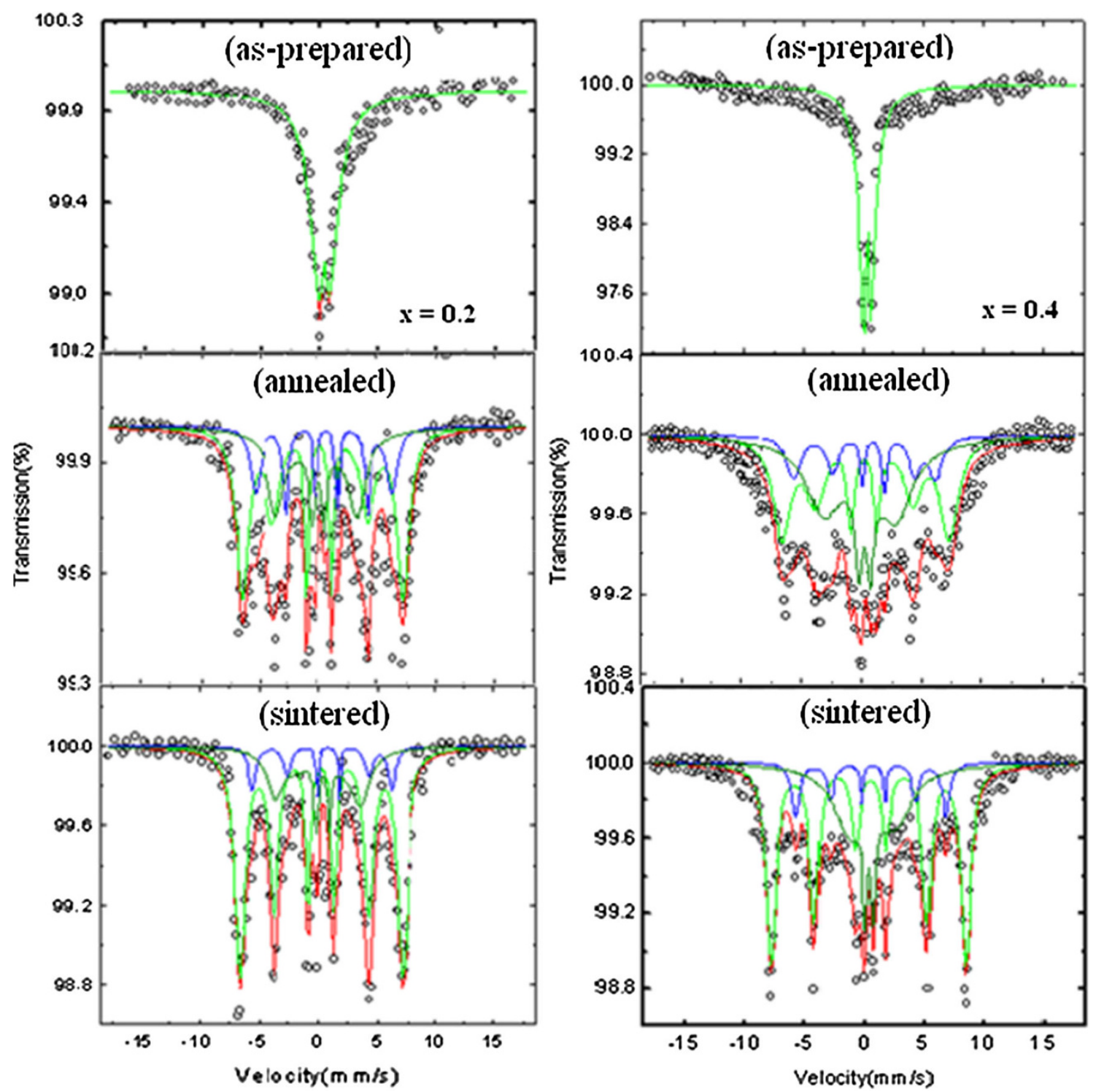

Fig. 3 Room temperature Mossbauer spectra of three specimens of $x=0.2$ and $x=0.4$ compositions of Mg-Mn ferrites

magnetization. Due to a distribution of energy barriers, some nanoparticles relax faster and the other slower at a given temperature. Consequently, the ferrimagnetic sextet and paramagnetic doublet appear simultaneously.

The Mossbauer spectra of sintered samples show two well-resolved Zeeman split sextets corresponding to the Aand $\mathrm{B}$ - sites $\mathrm{Fe}^{3+}$ ions, along with presence of central doublet. The presence of such central doublet for sintered samples is rather unexpected. This indicates a degree of short-range ordering within the predominantly long-range antiferromagnetic ordering. The increase in the population of the central doublet with increased diamagnetic substitution has been interpreted by Ishikawa (1964) as due to some $\mathrm{Fe}^{3+}$ ions being isolated from other magnetic ions giving rise to varying sizes of magnetic clusters which have little magnetic interaction with surroundings. Furthermore, magnetic ordering within the cluster is accompanied by much faster relaxation especially for smaller clusters and gives rise to the paramagnetic component.

The probability versus hyperfine magnetic field distribution curve, $p\left(H_{\mathrm{f}}\right)$, for a typical composition $x=0.2$ (annealed sample) is shown in Fig. 4. Attempt has been made to estimate particle size based on procedure suggested by Nikolaev et al. (2001). The idea of the method stems from the fact that, owing to the breaking of exchange bonds for surface atoms, their contribution to the total area bounded by the $p\left(H_{\mathrm{f}}\right)$ curve can be single out.

The size of the nanoparticles can be estimated by calculating the total area, $S=S_{\mathrm{e}}+S_{\mathrm{i}}$, that is bounded by the $p\left(H_{\mathrm{f}}\right)$ curve and by separating the part that corresponds to surface atoms, $S_{\mathrm{e}}$, and the contribution from the internal iron atoms $\left(S_{\mathrm{i}}\right)$. According to core-shell model (Tang et al. 




Fig. 4 Hyperfine field distribution function $p\left(H_{\mathrm{f}}\right)$ corresponding to the spectrum depicted in Fig. 3 (annealed sample of $x=0.2$ composition). Inset Schematic of core-shell structure

1991), nanoparticles can be thought of having core-shell structure with ferrimagnetically aligned core spins and a shell of finite and constant thickness made of weak magnetic and non-interacting mass i.e. magnetically dead layer. This means that the magnetic nature of the first crystalline layer on the nanoparticles is weakened by surface adsorption of non-magnetic ions but the magnetic properties of the core remain intact and resemble bulk magnetization (Tang et al. 1991). A more likely spin configuration in the shell is one in which the spins are almost random $(\sigma=0)$ at the surface and gradually recovers a collinear ferrimagnetic arrangement $\left(\sigma=\sigma_{\mathrm{s}}\right)$ with increasing distance from the surface (Muroi et al. 2001). Here, it is assumed that the volume of the external region [shell region having ring shape $\left.\left(V_{\mathrm{e}}\right)\right]$ is given by $V_{\mathrm{e}}=4 \pi r^{2} t$, and the volume of the internal region (core region having spherical shape) $\left(V_{\mathrm{i}}\right)$ is given by $V_{\mathrm{i}}=(4 / 3) \pi r^{3}$, where ' $r$ ' is the particle radius $=D / 2(D$ is the particle diameter $)$ and ' $t$ ' is the thickness of the surface layer. Since $S_{\mathrm{e}} / S_{\mathrm{i}}=V_{\mathrm{e}} / V_{\mathrm{i}}$ we can approximate that

$\frac{S_{\mathrm{e}}}{S_{\mathrm{i}}}=\frac{V_{\mathrm{e}}}{V_{\mathrm{i}}}=\frac{4 \pi(D / 2)^{2} \cdot t}{\frac{4}{3} \pi(D / 2)^{3}}=\frac{6 t}{D}$.

The term, $(6 t / D)$, plays very important role in correlating bulk to nanoparticles magnetization of ferrite systems, given by the relation $\sigma_{\mathrm{s}}{ }^{\text {nano }}=\sigma_{\mathrm{s}}{ }^{\text {bulk }}(1-(6 t / D))$ (Chen et al. 1996; Rangolia et al. 2008).

The shape of the spectrum at room temperature $\left(27^{\circ} \mathrm{C}\right)$ (Fig. 3) clearly shows a wide distribution of hyperfine magnetic field (HMF). The $p\left(H_{\mathrm{f}}\right)$ curve can be resolved into four broad peaks corresponding to four different values of hyperfine fields denoted by $H_{\mathrm{f} 1}, H_{\mathrm{f} 2}, H_{\mathrm{f} 3}$ and $H_{\mathrm{f} 4}$. The fields $H_{\mathrm{f} 1}$ and $H_{\mathrm{f} 2}$ correspond to iron ions in the shell region of a particle while $H_{\mathrm{f} 3}$ and $H_{\mathrm{f} 4}$ correspond to that of inner iron ions. Two reasons may account for the wider HMF distribution. Magnesium, manganese and iron ions might be statistically distributed among the available A- and $\mathrm{B}$-sites to give different kinds of environment to the iron nuclei resulting in broadening of $p\left(H_{\mathrm{f}}\right)$ distribution. The other possible reason could be the particle size distribution (Upadhyay 2003). The area under each peak has been used to estimate the volumes $V_{\mathrm{e}}$ and $V_{\mathrm{i}}$. In the present case we have found that $S_{\mathrm{e}} / S_{\mathrm{i}}=V_{\mathrm{e}} / V_{\mathrm{i}}=(6 t / D)=0.155$. It has been suggested by different workers that this shell layer can have thickness $(t)$ approximately equal to one lattice constant (Chen et al. 1996; Tang et al. 1991; Muroi et al. 2001). If we take $t=0.8448 \mathrm{~nm}$ (Table 1) determined from X-ray diffraction pattern analysis, we obtain $D \sim 33 \mathrm{~nm}$. The agreement between the particle size estimated with the help of the Mossbauer spectral analysis and TEM images/XRD patterns analysis (Table 1) can be regarded as quite satisfactory taking into account the approximating nature of the calculations.

\section{Conclusions}

Nanoparticles of $\mathrm{Mg}_{x} \mathrm{Mn}_{1-x} \mathrm{Fe}_{2} \mathrm{O}_{4}$ spinel ferrite system have been successfully synthesised by the chemical coprecipitation technique. We have established that the wet chemically prepared spinel ferrite compositions contain nanoparticles which give rise to the unusual magnetic properties to the system like superparamagnetism leading to the suppression of long-range magnetic ordering. The high temperature sintering of the wet prepared ferrites transforms them into the order magnetic structure of ceramic ferrites having long-range ferrimagnetic ordering. A method of estimating particle size by probability versus hyperfine magnetic field distribution data for nanocrystalline ferrite materials is found satisfactorily applicable.

Acknowledgments One of the authors (TKP) is thankful to Dr. Pooja U. Sharma for Mossbauer data analysis.

Open Access This article is distributed under the terms of the Creative Commons Attribution License which permits any use, distribution, and reproduction in any medium, provided the original author(s) and the source are credited.

\section{References}

Chen JP, Sorensen CM, Klabunde KJ, Hadjipanayis GC, Devlin E, Kostikas A (1996) Size dependant magnetic properties of $\mathrm{MnFe}_{2} \mathrm{O}_{4}$ fine particles synthesized by coprecipitation. Phys Rev B 54(13):9288-9296

Weast RC (ed) (1980-1981) CRC Handbook of Chemistry and Physics, 61st edn. CRC Press Inc., Florida, F-216 
Cullity BD (1978) Elements of X-ray diffraction. 2nd ed. AddisonWesley, Reading. 99

Gabriel JR, Ruby SL (1965) Computation of mossbauer spectra. Nucl Inst Meth 36:23-28

Gagan K, Kanthwal M, Chauhan BS, Sing M (2006) Cation distribution in mixed $\mathrm{Mg}-\mathrm{Mn}$ ferrite systems from X-ray diffraction technique and saturation magnetization. Ind J Pure Appl Phys 44:930-934

Ishikawa Y (1964) Superparamagnetism in magnetically dilute systems. J Appl Phys 35(3):1054-1059

Lakshman A, Subba Rao PSV, Rao BP, Rao KH (2005) Electrical properties of $\mathrm{In}^{3+}$ and $\mathrm{Cr}^{3+}$ substituted magnesium-manganese ferrites. J Phys D Appl Phys 38(5):673-678

Modi KB, Joshi HH, Kulkarni RG (1996) Magnetic and electrical properties of $\mathrm{Al}^{3+}$ substituted $\mathrm{MgFe}_{2} \mathrm{O}_{4}$. J Mater Sci 31:1311-1317

Modi KB, Vasoya NH, Lakhani VK, Pathak TK (2012) Spherical to Needle Shaped Particles Transformation Study on Nanocrystalline Mg-Mn Ferrites. J Adv Microsc Res 7(1):40-43

Muroi M, Street R, McCormiok PG, Amighian J (2001) Magnetic properties of ultrafine $\mathrm{MnFe}_{2} \mathrm{O}_{4}$ powders prepared by mechanochemical processing. Phys Rev B 63:184414-184420

Nikolaev VI, Shipilin AM, Zakharova IN (2001) On estimating nanoparticles size with the help of Mossbauer effect. Phys Solid State 43(8): 1455-1457

Pandya PB, Joshi HH, Kulkarni RG (1991) Bulk magnetic properties of $\mathrm{Co}-\mathrm{Zn}$ ferrites prepared by co-precipitation method. J Mater Sci 26:5509-5512

Parekh K, Upadhyay RV, Belova L, Rao KV (2006) Ternary monodispersed $\mathrm{Mn}_{0.5} \mathrm{Zn}_{0.5} \mathrm{Fe}_{2} \mathrm{O}_{4}$ ferrite nanoparticles: preparation and magnetic characterization. Nanotechnology 17:5970-5975

Pathak TK, Vaoya NH, Lakhani VK, Modi KB (2010) Structural and magnetic phase evolution study on needle shaped nanoparticles of magnesium ferrites. Ceram Int 36(1):275-281

Rangolia MK, Chhantbar MC, Tanna AR, Modi KB, Baldha GJ, Joshi $\mathrm{HH}$ (2008) Magnetic behaviour of nano-sized and coarse powders of $\mathrm{Cd}-\mathrm{Ni}$ ferrites synthesized by wet- chemical route. Ind J Pure Appl Phys 46:60-64

Sharma RK, Suwalka O, Lakshmi N, Venugopalan K, Banerjee A, Joy PA (2005) Synthesis of Chromium substituted nanoparticles of cobalt zinc ferrites by coprecipitation. Mater Lett 59(27):3402-3405

Singhal S, Jauhar S, Singh J, Chandra K, Bansal S (2012) Investigation of structural, magnetic, electrical and Optical properties of chromium substituted cobalt ferrites $\left(\mathrm{CoCr}_{\mathrm{x}}\right.$ $\left.\mathrm{Fe}_{2-\mathrm{x}} \mathrm{O}_{4}, 0 \leq x \leq 1\right)$ synthesized using sol gel auto combustion method. J Mol Struct 1012:182-188

Tang ZX, Sorensen CM, Klabunde KJ, Hadjipanayis GC (1991) Sizedependent Curie temperature in nanoscale $\mathrm{MnFe}_{2} \mathrm{O}_{4}$ particles. Phys Rev Lett 67(25):3602-3605

Upadhyay C (2003) Controlled formation of nanosize spinel ferrites $(4-15 \mathrm{~nm})$ and their magnetic and structural studies. Ph.D. thesis, IIT-Kanpur, India. 102

Vasoya NH, Vanpariya LH, Sakariya PN, Timbadiya MD, Pathak TK, Lakhani VK, Modi KB (2010) Synthesis of nanostructured material by mechanical milling and study on structural property modification in $\mathrm{Ni}_{0.5} \mathrm{Zn}_{0.5} \mathrm{Fe}_{2} \mathrm{O}_{4}$. Ceram Int 36(3):947-954 